\title{
OSCILLATIONS IN A MOLECULAR STRUCTURED CELL POPULATION MODEL
}

\author{
RICARDO BORGES, ÀNGEL CALSINA, SÍLVIA CUADRADO
}

\begin{abstract}
We consider a nonlinear cyclin content structured model of a cell population divided into proliferative and quiescent cells. We show, for particular values of the parameters, existence of solutions that do not depend on the cyclin content. We make numerical simulations for the general case obtaining, for some values of the parameters convergence to the steady state but also oscillations of the population for others.
\end{abstract}

\section{INTRODUCTION}

The study of cell populations by means of structured models can be traced back to the works of Von Foerster [14] and later on of Bell and Anderson [8]. Since then, many researchers have contributed to this field (see for instance [13], [4] , 5], 2]). Cell structured population models with proliferating and quiescent compartments have also been extensively studied (see for instance [12], [17, [11]).

In [6] and [7] a nonlinear cell population model for both, tumoral and healthy tissue is introduced in which cells are structured with respect to age and with respect to the content of a group of proteins called cyclin and CDK (cyclin dependent kinases) which plays an important role in the regulation of the cell cycle (see [15]). Cells are divided into two categories: proliferating cells which grow and divide, giving birth at the end of the cycle to new cells, or else transit to the quiescent compartment, and quiescent cells which do not age, nor divide nor change their cyclin content. They may either go back to the proliferating compartment or stay in the quiescent one. Under suitable hypotheses, the authors prove exponential growth of the solutions in the tumoral case and boundedness of the solutions in the case of healthy tissue. In the second case they show numerical evidence of convergence to a nontrivial equilibrium in all the cases they study. A similar cell population model structured with respect to a certain protein content is analyzed in 10 .

In [9] we considered the age independent version of the model considered in [6] and [7], that is, the following first order nonlinear partial differential equations

This work was partially supported by the projects MTM2008-06349-C03-03 DGI - MCI (Spain) and 2009-SGR-345 from Agaur (Generalitat de Catalunya). 
system with nonlocal terms structured only with respect to the cyclin content

$$
\left\{\begin{aligned}
\frac{\partial}{\partial t} p(x, t)+\frac{\partial}{\partial x}(\Gamma(x) p(x, t))= & -\left[L(x)+F(x)+d_{1}\right] p(x, t) \\
& +G(N(t)) q(x, t)+2 \int_{x}^{x_{M}} \frac{F(y)}{y} p(y, t) d y, \\
\frac{\partial}{\partial t} q(x, t) & =L(x) p(x, t)-\left[G(N(t))+d_{2}\right] q(x, t)
\end{aligned}\right.
$$

where $p(x, t)$ and $q(x, t)$ are the density of proliferating and quiescent cells (respectively) at time $t$ with respect to the cyclin content $x$ (see Section 2 for a full description of the model). We proved the existence and uniqueness of a steady state for this model in the case of healthy tissue.

In the present paper we are interested in the asymptotic behavior of the time dependent solutions. We start by, in Section 3, proving the existence, for some values of the parameters, of solutions of System (11) that do not depend on the cyclin content and hence satisfy an ordinary differential equations system. We analyze the complete asymptotic behavior of this ordinary differential equations system showing that the unique nontrivial steady state (when it exists) is asymptotically stable under some conditions and unstable when the reverse conditions hold. The instability appears through a Hopf bifurcation which leads to the existence of stable self-sustained oscillations of the populations. In Section 4 we use a numerical scheme to illustrate the possible asymptotic behaviors of System (1). We obtain, depending on the values of the parameters, existence of stable and unstable equilibria as well as stable limit cycles. The numerical scheme is based on a discretization of System (1) by means of a time invariant grid, called the natural grid (see [3], [16], [1]) which is obtained by an explicit integration of the characteristic equation.

The approximate solution is computed by means of a predictor-corrector method which numerically integrates the system along the characteristics. It also involves the numerical computations of integrals corresponding to the non local term in the first equation in System (11). In contrast to [3], where the nonlocal term just occurs in the boundary condition, we have to approximate the integral value at any point of the grid. To avoid algorithmic complication we use a trapezoidal quadrature rule (of second order accuracy). This explains the use of a second order Runge-Kutta method and not a higher order one. The presence of this non local term in System (1), modeling unequal cell division, is also the main difference with respect to the model in [1] from the point of view of numerical requirements.

Finally, in the Appendix we have performed two tests for the numerical scheme, the first one comparing the numerical solution to an exact solution for a simplified version of System (11) without non local terms, and the second one comparing the numerical solutions of System (11) in the case of $x$-independent solutions with the solutions of the corresponding ordinary differential system. 


\section{THE MODEL}

We consider the following first order nonlinear (nonlocal) partial differential equations system

$$
\left\{\begin{aligned}
\frac{\partial}{\partial t} p(x, t)+\frac{\partial}{\partial x}(\Gamma(x) p(x, t))= & -\left[L(x)+F(x)+d_{1}\right] p(x, t) \\
& +G(N(t)) q(x, t)+2 \int_{x}^{x_{M}} \frac{F(y)}{y} p(y, t) d y \\
\frac{\partial}{\partial t} q(x, t)= & L(x) p(x, t)-\left[G(N(t))+d_{2}\right] q(x, t)
\end{aligned}\right.
$$

where $p(x, t)$ and $q(x, t)$ are the density of proliferating and quiescent cells (respectively) at time $t$ with respect to the content of cyclin $x \in\left(0, x_{M}\right)$. Cells leave the proliferating stage because of apoptosis (programmed cell death) that is assumed to occur at a rate $d_{1}$, because of cell division with rate $F(x)$ or because of transition to the quiescent stage that occurs according to a "leak" function $L(x)$.

We denote the total weighted population by

$$
N(t)=N(p(x, t), q(x, t)):=\int_{0}^{x_{M}} \phi(x) p(x, t)+\psi(x) q(x, t) d x
$$

where $\phi$ and $\psi$ are positive bounded functions. Cells can leave the quiescent stage because of apoptosis, that is assumed to occur at a rate $d_{2}$ or because of transition back to the proliferating stage that is assumed to occur according to a "recruitment" function $G$ which is assumed to be a smooth strictly decreasing function of the total weighted population, satisfying $G(0)>0$ and that $G$ tends to 0 when $N$ goes to infinity (case of healthy tissue).

We assume that $G, L$ and $F$ are bounded, positive and continuous functions and that $\frac{F(x)}{x}$ has a finite positive limit when $x$ goes to 0 (implying in particular $F(0)=0) . \Gamma(x)$ represents the evolution speed of cyclin content with respect to time. We assume that $\Gamma \in C^{2}\left[0, x_{M}\right], \Gamma(0)=\Gamma\left(x_{M}\right)=0, \Gamma(x)>0$ for all $x \in\left(0, x_{M}\right)$ and the lateral derivatives satisfy $\Gamma^{\prime}(0)>0, \Gamma^{\prime}\left(x_{M}\right)<0$.

\section{3. $x$-INDEPENDENT SOLUTIONS}

In this section we look for solutions of System (2) that do not depend on the cyclin content $x$, that is, solutions of the form $(p(t), q(t))$. From the second equation in (2) we obtain that, in order to have solutions that are independent of $x$ we must impose that the leak function is constant, that is, we must assume $L(x)=L_{0}>0$. From the first equation in (2) we then have that an $x$-independent solution $(p(t), q(t))$ of (2) must satisfy the equality

$$
\dot{p}(t)+\Gamma^{\prime}(x) p(t)=-\left(L_{0}+F(x)+d_{1}\right) p(t)+2 p(t) \int_{x}^{x_{M}} \frac{F(y)}{y} d y+G(N(t)) q(t),
$$


or, equivalently

$$
\dot{p}(t)=-\left(L_{0}+F(x)+\Gamma^{\prime}(x)+d_{1}-2 \int_{x}^{x_{M}} \frac{F(y)}{y} d y\right) p(t)+G(N(t)) q(t),
$$

which implies that

$$
L_{0}+F(x)+\Gamma^{\prime}(x)+d_{1}-2 \int_{x}^{x_{M}} \frac{F(y)}{y} d y
$$

should be constant. Deriving, we have that the equality

$$
F^{\prime}(x)+\Gamma^{\prime \prime}(x)+2 \frac{F(x)}{x}=0
$$

must hold. Solving (44) and using that $\lim _{x \rightarrow 0} \frac{F(x)}{x}=: b<\infty$ we have that

$$
F(x)=-\frac{1}{x^{2}} \int_{0}^{x} s^{2} \Gamma^{\prime \prime}(s) \mathrm{d} s .
$$

Substituting (5) in (3) and avaluating at $x=x_{M}$ we have that

$$
L_{0}+F(x)+\Gamma^{\prime}(x)+d_{1}-2 \int_{x}^{x_{M}} \frac{F(y)}{y} d y=L_{0}+d_{1}-A
$$

with

$$
A=\frac{1}{x_{M}^{2}} \int_{0}^{x_{M}} s^{2} \Gamma^{\prime \prime}(s) \mathrm{d} s-\Gamma^{\prime}\left(x_{M}\right) .
$$

Notice that, integrating the above equation on the interval $\left(0, x_{M}\right)$ we also get $A=\frac{1}{x_{M}} \int_{0}^{x_{M}} F(x) \mathrm{d} x$.

So, System (21) has $x$-independent solutions $(p(t), q(t))$ if $L(x)$ is a constant $L_{0}$ and (5) holds. Conversely, if the same conditions hold and $(p(t), q(t))$ satisfis the ordinary differential equations system

$$
\left\{\begin{array}{l}
\dot{p}=\left(A-L_{0}-d_{1}\right) p+G(N(t)) q, \\
\dot{q}=L_{0} p-\left(d_{2}+G(N(t)) q,\right.
\end{array}\right.
$$

where $N:=N(p, q)=p \int_{0}^{x_{M}} \phi(x) d x+q \int_{0}^{x_{M}} \psi(x) d x=: k_{1} p+k_{2} q$ with $k_{1}, k_{2}$ positive real numbers and $A$ given by (6) $)$, then $(p(t), q(t))$ is an $x$-independent solution of System (2).

3.1. Equilibria. Let us now study the existence of equilibria of System (7) (which will imply the existence of $x$-independent equilibria of System (2) under the conditions above).

Proposition 1. System (7) has a unique non trivial equilibrium solution if and only if the inequalities

$$
\frac{A}{d_{1}+L_{0}}<1<\frac{A}{d_{1}+L_{0} \frac{d_{2}}{d_{2}+G(0)}}
$$

hold. 
Proof. A non trivial equilibrium solution $(\hat{p}, \hat{q})$ of System (7) satisfies:

$$
\begin{aligned}
& 0=\left(A-L_{0}-d_{1}\right) \hat{p}+G(\hat{N}) \hat{q} \\
& 0=L_{0} \hat{p}-\left(G(\hat{N})+d_{2}\right) \hat{q}
\end{aligned}
$$

that is, $\hat{p}=\frac{G(\hat{N}) \hat{q}}{L_{0}+d_{1}-A}=\frac{\left(d_{2}+G(\hat{N})\right) \hat{q}}{L_{0}}$, where $\hat{N}=k_{1} \hat{p}+k_{2} \hat{q}$.

Since $\hat{q} \neq 0$ this is equivalent to

$$
G(\hat{N})=\frac{\left(L_{0}+d_{1}-A\right) d_{2}}{A-d_{1}}
$$

Since $\mathrm{G}$ is an strictly decreasing function that tends to zero then $N$ tends to infinity, there will be a unique solution, $\hat{N}$ of (9) (and therefore a unique nontrivial equilibrium point) if and only if

$$
0<\frac{\left(L_{0}+d_{1}-A\right) d_{2}}{A-d_{1}}<G(0)
$$

And easy computation shows that the previous inequalities are equivalent to (8).

Remark 1. If we think of system (7) as a model for the dynamics of a population with two groups of individuals where $A$ denotes the per capita birth rate of the first group, $d_{1}$ and $d_{2}$ the mortality rates and $L_{0}$ and $G(N)$ the transition rates between the two groups, then the inequalities (8) can be interpreted using the concept of the expected number of offspring of an individual in its lifespan $R_{0}$ assuming a constant value of the "interaction" variable $N$. Indeed, let us compute $R_{0}$ for this model.

Let $X$ be a random variable denoting the number of offspring in the lifespan of an individual and $Z$ be a random variable taking the value 0 if an individual taken at random does not go to the quiescent stage, 1 if it goes once to the quiescent stage and returns back to the proliferating one, and so on. Note that $Z=0$ is the event that an exponentially distributed random variable with expected value $\frac{1}{L_{0}}$ takes a value larger than another independent exponentially distributed random variable with expected value $\frac{1}{d_{1}}$ and therefore $P(Z=0)=\frac{d_{1}}{d_{1}+L_{0}}$. In the same way we obtain that $P(Z=1)=\frac{L_{0}}{d_{1}+L_{0}} \frac{G(N)}{d_{2}+G(N)} \frac{d_{1}}{d_{1}+L_{0}}, P(Z=2)=$ $\left(\frac{L_{0}}{d_{1}+L_{0}} \frac{G(N)}{d_{2}+G(N)}\right)^{2} \frac{d_{1}}{d_{1}+L_{0}}, \ldots, P(Z=k)=\left(\frac{P(Z=1)}{P(Z=0)}\right)^{k} P(Z=0)$. 
Then

$$
\begin{aligned}
R_{0}(N) & =E(X)=\sum_{k=0}^{\infty} E(X \mid Z=k) P(Z=k) \\
& =E(X \mid Z=0) P(Z=0) \sum_{k=0}^{\infty}\left(\frac{P(Z=1)}{P(Z=0)}\right)^{k} \\
& =\frac{A}{d_{1}}\left(\frac{d_{1}}{d_{1}+L_{0}}\right) \sum_{k=0}^{\infty}\left(\frac{L_{0}}{d_{1}+L_{0}} \frac{G(N)}{d_{2}+G(N)}\right)^{k} \\
& =\left(\frac{A}{d_{1}+L_{0}}\right)\left(\frac{1}{1-\frac{L_{0} G(N)}{\left(d_{1}+L_{0}\right)\left(d_{2}+G(N)\right)}}\right) \\
& =\frac{A}{d_{1}+L_{0} \frac{d_{2}}{d_{2}+G(N)}}
\end{aligned}
$$

where we have used that $E(X \mid Z=k)=E(X \mid Z=0)=\frac{A}{d_{1}}$ since System (7) assumes that the second group of individuals do not reproduce, $\frac{1}{d_{1}}$ is the expected lifetime of a reproducing individual and $A$ the per capita and time unit fertility.

The inequalities (8) in Proposition 1 correspond to assuming $R_{0}(0)>1$ and $R_{0}(\infty)<1$ (recall that $G(\infty)=0$ ).

\subsection{Asymptotic behavior.}

Proposition 2. Let us assume that $\frac{A}{d_{1}+L_{0} \frac{d_{2}}{d_{2}+G(0)}} \leq 1$. Then the trivial equilibrium is a global attractor of System (7).

Proof. First notice that if $A \leq d_{1}$ then $(p+q) \cdot \leq 0$ and the claim follows. Now let us assume $A>d_{1}$ and notice that by hypothesis $L_{0}+d_{1}-A>0$.

By the implicit function theorem, the isoclines of System (7) define functions $p=F_{1}(q)$ and $p=F_{2}(q)$ respectively through the relations

$$
p=\frac{G(N(p, q)) q}{L_{0}+d_{1}-A} \text { and } p=\frac{\left(d_{2}+G(N(p, q))\right) q}{L_{0}} .
$$

We obviously have $F_{1}(0)=F_{2}(0)=0$. Moreover, for all positive $q$ we have that $F_{1}(q)<F_{2}(q)$. Indeed, since $\frac{A}{d_{1}+L_{0} \frac{d_{2}}{d_{2}+G(0)}} \leq 1$ or, equivalently $\left(L_{0}+d_{1}-A\right) d_{2}-$ $G(0)\left(A-d_{1}\right) \geq 0, G$ is strictly decreasing and $A>d_{1}$ we have

$$
\left(L_{0}+d_{1}-A\right) d_{2}-G(N)\left(A-d_{1}\right)>0
$$

for all $N>0$. 
Let us now assume that there exists a positive $\hat{q}$ such that $\hat{p}_{2}:=F_{2}(\hat{q}) \leq F_{1}(\hat{q})=$ : $\hat{p}_{1}$. We will have

$$
\hat{p}_{2}=\frac{\left(d_{2}+G\left(N\left(\hat{p}_{2}, \hat{q}\right)\right)\right) \hat{q}}{L_{0}} \leq \hat{p}_{1}=\frac{G\left(N\left(\hat{p}_{1}, \hat{q}\right)\right) \hat{q}}{L_{0}+d_{1}-A} .
$$

Hence

$$
\left(L_{0}+d_{1}-A\right) d_{2}-G\left(N\left(\hat{p}_{2}, \hat{q}\right)\right)\left(A-d_{1}\right) \leq L_{0}\left(G\left(N\left(\hat{p}_{1}, \hat{q}\right)\right)-G\left(N\left(\hat{p}_{2}, \hat{q}\right)\right)\right) \leq 0
$$

since $\hat{p}_{2} \leq \hat{p}_{1}, N$ is increasing as function of $p$ and $G$ is decreasing; a contradiction. Now notice that for any $q_{0}$, the regions $\left\{(p, q): q \leq q_{0}\right.$ and $\left.p \leq F_{2}(q)\right\}$ are positively invariant and that any trajectory eventually enters some of them since $\lim _{q \rightarrow \infty} F_{2}(q)=\infty$ and $\dot{p}(t)<0$ if $p(t)>F_{2}(q(t))$. These bounded regions cannot contain periodic orbits due to the direction of the vectorial field on the isocline lines. The statement about asymptotic behavior follows from the Bendixson Poincaré theorem.

Proposition 3. Let us assume that $\frac{A}{d_{1}+L_{0}} \geq 1$. Then all the trajectories of System (7) are unbounded.

Proof. Under this hypothesis only the isocline of horizontal vectorial field remains in the open first quadrant. The direction of the vectorial field in the first quadrant gives the statement.

Remark 2. As before, the results can be interpreted in terms of $R_{0}$. The assumption in Proposition 2 is that $R_{0}<1$ in ideal conditions (zero population number) ensuring extinction. The assumption in Proposition 3 is that $R_{0}>1$ in starvation conditions (infinite population number) giving rise to unbounded population.

The two previous propositions give us the behavior of System (17) when there is not nontrivial steady state. Under the hypotheses of Proposition 1, System (7) has a unique non trivial steady state that can be written

$$
(\hat{p}, \hat{q})=\left(\frac{\hat{N} d_{2}}{\left(A-d_{1}\right) k_{2}+d_{2} k_{1}}, \frac{\hat{N}\left(A-d_{1}\right)}{\left(A-d_{1}\right) k_{2}+d_{2} k_{1}}\right)
$$

where $\hat{N}$ is the unique solution of $G(\hat{N})=\frac{\left(L_{0}+d_{1}-A\right) d_{2}}{A-d_{1}}$. Indeed, using (10) and (9) we have $\hat{p}=\frac{d_{2}}{A-d_{1}} \hat{q}$ and so $\hat{N}=k_{1} \hat{p}+k_{2} \hat{q}=\left(k_{1} \frac{d_{2}}{A-d_{1}}+k_{2}\right) \hat{q}$ which clearly implies the claim.

Theorem 3.1. Under the hypotheses of Proposition 1 the unique non trivial steady state $(\hat{p}, \hat{q})$ of System (7) is (locally) asymptotically stable whenever $\left(A-d_{1}-L_{0}-d_{2}-G(\hat{N})+\hat{q} G^{\prime}(\hat{N})\left(k_{1}-k_{2}\right)\right)<0$ and it is unstable if the reverse strict inequality holds. In particular it is asymptotically stable if $k_{1} \geq k_{2}$. 
Proof. The Jacobian matrix of System (7) at the steady state is given by

$$
J(\hat{p}, \hat{q})=\left(\begin{array}{cc}
A-d_{1}-L_{0}+k_{1} G^{\prime}(\hat{N}) \hat{q} & \hat{q} k_{2} G^{\prime}(\hat{N})+G(\hat{N}) \\
L_{0}-\hat{q} k_{1} G^{\prime}(\hat{N}) & -\hat{q} k_{2} G^{\prime}(\hat{N})-\left(d_{2}+G(\hat{N})\right) .
\end{array}\right)
$$

Denoting by $\lambda_{1}$ and $\lambda_{2}$ the two eigenvalues of $J(\hat{p}, \hat{q})$ and using (9) we have that

$$
\lambda_{1} \lambda_{2}=\hat{q} G^{\prime}(\hat{N})\left(k_{2}\left(d_{1}-A\right)-k_{1} d_{2}\right) .
$$

Under the hypotheses of Proposition 1 which imply $A>d_{1}$ and since $G^{\prime}(\hat{N})<0$ we then always have that $\lambda_{1} \lambda_{2}>0$. On the other hand

$$
\lambda_{1}+\lambda_{2}=A-d_{1}-L_{0}-d_{2}-G(\hat{N})+\hat{q} G^{\prime}(\hat{N})\left(k_{1}-k_{2}\right),
$$

giving the first statement. Assuming $k_{1} \geq k_{2}$ we obtain using (8) , that $\lambda_{1}+\lambda_{2}<0$ which gives the second statement.

Since the previous theorem reduces the study of the stability of the non trivial steady state to the study of the sign of the trace of the Jacobian matrix, in the following we choose particular forms of the function $G$ and find reasonable values of the parameters that make the trace negative and so give instability.

Let us take $G(N)=G(0) e^{-c N}$ and assume $k_{2}>k_{1}$. Then using that $G(\hat{N})=$ $\frac{\left(L_{0}+d_{1}-A\right) d_{2}}{A-d_{1}}, \hat{q}=\frac{\hat{N}\left(A-d_{1}\right)}{\left(A-d_{1}\right) k_{2}+d_{2} k_{1}}$ and denoting by $A_{1}:=A-d_{1}$ we have

$$
\begin{aligned}
\lambda_{1}+\lambda_{2} & =A_{1}-L_{0}-d_{2}-\frac{\left(L_{0}-A_{1}\right) d_{2}}{A_{1}}+\frac{\hat{N} A_{1}}{A_{1} k_{2}+d_{2} k_{1}}\left(-c \frac{\left(L_{0}-A_{1}\right) d_{2}}{A_{1}}\right)\left(k_{1}-k_{2}\right) \\
& =A_{1}-L_{0}-d_{2}-\frac{\left(L_{0}-A_{1}\right) d_{2}}{A_{1}}-\frac{\ln \left(\frac{G(0)}{\left.\frac{\left(L_{0}-A_{1}\right) d_{2}}{A_{1}}\right) A_{1}}\right.}{A_{1} k_{2}+d_{2} k_{1}}\left(\frac{\left(L_{0}-A_{1}\right) d_{2}}{A_{1}}\right)\left(k_{1}-k_{2}\right)
\end{aligned}
$$

We can see then that $\lambda_{1}+\lambda_{2}<0$ is equivalent to

$$
\begin{aligned}
\ln (G(0)) & <\ln \left(\frac{\left(L_{0}-A_{1}\right) d_{2}}{A_{1}}\right)+\frac{\frac{A_{1}}{d_{2}}+\frac{L_{0}}{L_{0}-A_{1}}}{k-1}\left(k+\frac{d_{2}}{A_{1}}\right) \\
& =: H\left(k, L_{0}, A_{1}, d_{2}\right)
\end{aligned}
$$

where we have denoted by $k=\frac{k_{2}}{k_{1}}$ (assuming $k_{1}>0$ ). Then, a necessary and sufficient condition for the eigenvalues to have negative real part is

$$
G(0)<e^{H\left(k, L_{0}, A_{1}, d_{2}\right)} .
$$

Whenever $G(0)=e^{H\left(k, L_{0}, A_{1}, d_{2}\right)}$ we will have purely imaginary eigenvalues. Considering $H$ as a function of $A_{1}$ and $d_{2}$ it can be seen that the infimum value of $H$ is

$$
\lim _{A_{1} \rightarrow 0} H\left(k, L_{0}, A_{1}, A_{1}\right)=\ln \left(L_{0}\right)+2\left(\frac{k+1}{k-1}\right) .
$$


Indeed, let us consider, for fixed $k$ and $L_{0}$, the function

$$
h(u, v)=\ln v+\ln u+\left(\frac{1}{u}+\frac{L_{0}}{v}\right) \frac{k+u}{k-1}
$$

defined on $\mathbb{R}^{2+}$ and notice $H\left(k, L_{0}, A_{1}, d_{2}\right)=h\left(\frac{d_{2}}{A_{1}}, L_{0}-A_{1}\right)$. Then we will have

$$
\begin{aligned}
\inf _{\left\{\left(A_{1}, d_{2}\right) \in \mathbb{R}^{2+}: A_{1}<L_{0}\right\}} H\left(k, L_{0}, A_{1}, d_{2}\right) & =\inf _{\left\{\left(A_{1}, d_{2}\right) \in \mathbb{R}^{2+}: A_{1}<L_{0}\right\}} h\left(\frac{d_{2}}{A_{1}}, L_{0}-A_{1}\right) \\
& =\inf _{\left\{(u, v) \in \mathbb{R}^{2+}: v<L_{0}\right\}} h(u, v) \\
& =h\left(1, L_{0}\right)=\ln L_{0}+2 \frac{k+1}{k-1},
\end{aligned}
$$

where the last but one equality is proven as follows. The function $h$ is of class $C^{1}$ on $R:=\left\{(u, v) \in \mathbb{R}^{2+}: v<L_{0}\right\}$ with limit equal to infinity at any point of the two coordinate axes as well as when $u$ tends to infinity, with no critical points in $R$ since $\frac{\partial h}{\partial v}(u, v)=\frac{1}{v^{2}}\left(v-L_{0} \frac{k+u}{k-1}\right)<0$ for $v<L_{0}$ and such that has a (unique) minimum point on the remaining part of the boundary of $R\left(v=L_{0}\right.$, i.e., $\left.A_{1}=0\right)$ at $u=1\left(d_{2}=A_{1}\right)$ since $\frac{\partial h}{\partial u}\left(u, L_{0}\right)=\frac{(u+k)(u-1)}{(k-1) u^{2}}$.

From (12) and (13) we have that, in particular, a sufficient condition for stability is

$$
G(0)<L_{0} e^{2\left(\frac{k+1}{k-1}\right)} .
$$

Moreover, if $A_{1}=d_{2}$ and they are small enough, then the reverse inequality gives instability. We use this to find moderate values of the parameters that lead to instability of the steady state giving rise to a limit cycle as can be seen in Figure 1 . The case $k_{1}=0$ can be analyzed in the same way.

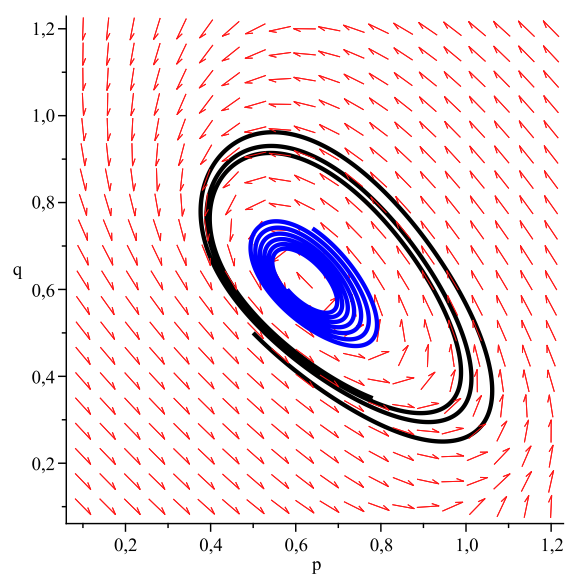

FIGURE 1. Illustration with $k=6, A_{1}=d_{2}=0.1, L_{0}=$ $0.2, G(0)=7.5$. Notice $G(0)=7.5>e^{H(6,0.2,0.1,0.1)}=6.67$. 
In a similar way, taking $G(N)=\frac{G(0)}{1+N^{n}}$ (which is the nonlinearity considered in [6] and [7]) we can find values of the parameters that also lead to instability of the steady state going to a limit cycle as can be seen in Figure 2.

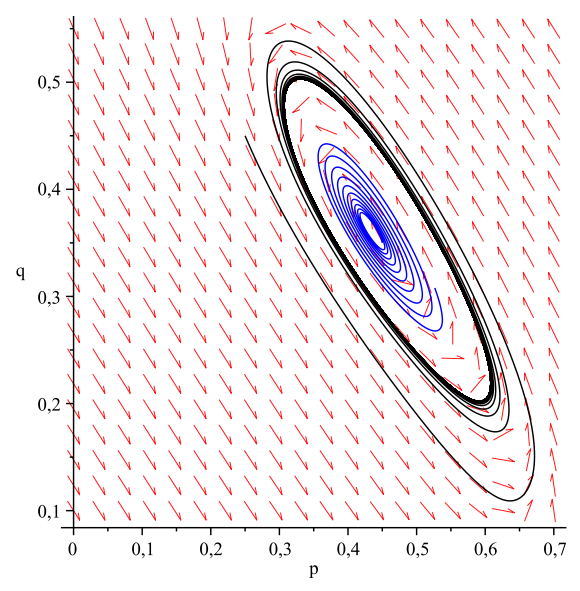

FiguRE 2. Illustration with $k=4, n=4, A_{1}=0.1, d_{2}=0.12$, $L_{0}=0.5, G(0)=6.5$.

\section{NUMERICAL SIMULATION}

We now go back to the full problem (2) and describe a numerical scheme and the numerical results obtained by using it. In particular we emphasize the asymptotic behavior of $x$-dependent solutions, showing the existence of stable and unstable equilibria, as well as stable limit cycles, depending on the different values of the parameters. To begin with, following the lines of [3], we build up the so-called natural grid, i.e. a grid such that, in the case of a single equation, which is the situation in

[3], consists of a set of points $\left\{\left(x_{i}, t_{j}\right):-1 \leq i \leq n+1,0 \leq j \leq m\right\}$ in such a way that $\left(x_{i-1}, t_{j-1}\right)$ and $\left(x_{i}, t_{j}\right)$ belong to the same characteristic line. Notice that (2) is a system of two first order partial differential equations and consequently, it has two families of (base) characteristic lines. This fact in general would complicate the build up of a natural grid, which should be such that the points of the grid are intersection points of characteristics of the two families.

Fortunately, the equations in (2) are autonomous and moreover, the characteristics of the second one are straight vertical lines in the plane $(x, t)$. This allows to extend the procedure of [3] to our case, and we shall obtain a rectangular grid (with edges parallel to the coordinate axes) with uniform time step size and nonuniform $x$ step size. On the other hand, since $\Gamma$ vanishes at both ends of the interval $\left(0, x_{M}\right), x=0$ and $x=x_{M}$ will be characteristic lines (for both equations) whereas the other characteristics (of the first equation) will not cross the ends of the interval. 
Let us call $\Psi(t ; s, x)$ the solution of the initial value problem for the characteristic equation

$$
z^{\prime}(t)=\Gamma(z(t)), \quad z(s)=x,
$$

and let us also define $\Phi(t, x)=\Psi(t ; 0, x)$. Unlike [3] we assume that this equation can be solved explicitly and so we have the exact solution. Indeed, our computations are all based in taking $x_{M}=1$ and $\Gamma(x)=x(1-x)$. We begin by choosing an arbitrary (small) positive number $x_{0}$ and compute $T$ such that $\Phi\left(T, x_{0}\right)=1-x_{0}$. Then we take $n=\left[T \max (\Gamma) / x_{0}\right]+1$ (here [ ] stands for the integer part), the time step size $h=T / n$, a natural number $m \geq n$ and the grid defined by:

$G=\left\{\left(x_{i}, t_{j}\right): t_{j}=j h, j=0, \ldots, m, x_{i}=\Phi\left(t_{i}, x_{0}\right), i=0, \ldots, n, x_{-1}=0, x_{n+1}=1\right\}$.

and notice that

$$
x_{i}=\Phi\left(t_{i}, x_{0}\right)=\Phi\left(h, \Phi\left(t_{i-1}, x_{0}\right)\right)=\Phi\left(h, x_{i-1}\right)
$$

and so $x_{i}-x_{i-1} \leq \max (\Gamma) h \leq x_{0}$. Hence, the size of the $x$ steps is bounded by $x_{0}$.

We will use this grid to compute an approximate solution of the initial value problem for (2), with initial conditions $p(x, t)=p^{0}(x)$ and $q(x, t)=q^{0}(x)$.

Now we explain how to compute the approximate solution at time $t_{j}$ given an approximate solution till time $t_{j-1}$. Let us assume that we already have an approximate solution up to time step $j-1$ (for some $j \geq 1$ ) given by the values $\left(p_{i}^{j-1}, q_{i}^{j-1}\right)$, at the points $\left(x_{i}, t_{j-1}\right), i=-1, \ldots, n+1$, taking into account that $p_{i}^{0}=p^{0}\left(x_{i}\right), \quad q_{i}^{0}=q^{0}\left(x_{i}\right)$, and let us assume that $(p(x, t), q(x, t))$ is an exact solution to (2) such that $p\left(x_{i}, t_{j-1}\right)=p_{i}^{j-1}$ and $q\left(x_{i}, t_{j-1}\right)=q_{i}^{j-1}$. Let us also call $S(x, t)=2 \int_{x}^{1} \frac{F(y)}{y} p(y, t) d y$ and $N(t):=\int_{0}^{1} \phi(x) p(x, t)+\psi(x) q(x, t) d x$.

Now we define, for $i=1, \ldots, n, P_{i}(t)=p\left(\Psi\left(t ; t_{j-1}, x_{i-1}\right), t\right)$ and, for $i=$ $-1, \ldots, n+1, Q_{i}(t)=q\left(x_{i}, t\right)$. We also define $f(x, p, q, S, N):=-\left[\Gamma^{\prime}(x)+L(x)+\right.$ $\left.F(x)+d_{1}\right] p+G(N) q+S$ and $g(x, p, q, N):=L(x) p-\left(G(N)+d_{2}\right) q$. We will have, for $i=1, \ldots, n$, the following ordinary differential equations

$$
\begin{aligned}
\frac{d P_{i}}{d t}(t) & =p_{t}\left(\Psi\left(t ; t_{j-1}, x_{i-1}\right), t\right)+p_{x}\left(\Psi\left(t ; t_{j-1}, x_{i-1}\right), t\right) \Psi_{t}\left(t ; t_{j-1}, x_{i-1}\right) \\
& =p_{t}\left(\Psi\left(t ; t_{j-1}, x_{i-1}\right), t\right)+\Gamma\left(\Psi\left(t ; t_{j-1}, x_{i-1}\right)\right) p_{x}\left(\Psi\left(t ; t_{j-1}, x_{i-1}\right), t\right) \\
& =f\left(\Psi\left(t ; t_{j-1}, x_{i-1}\right), P_{i}(t), q\left(\Psi\left(t ; t_{j-1}, x_{i-1}\right), t\right),\right. \\
& \left.S\left(\Psi\left(t ; t_{j-1}, x_{i-1}\right), t\right), N(t)\right), \\
P_{i}\left(t_{j-1}\right) & =p\left(x_{i-1}, t_{j-1}\right)=p_{i-1}^{j-1},
\end{aligned}
$$


and, for $i=-1, \ldots, n+1$,

$$
\begin{aligned}
\frac{d Q_{i}}{d t}(t) & =g\left(x_{i}, p\left(x_{i}, t\right), q\left(x_{i}, t\right), N(t)\right), \\
Q_{i}\left(t_{j-1}\right) & =q\left(x_{i}, t_{j-1}\right)=q_{i}^{j-1} .
\end{aligned}
$$

Notice that the boundary values of $P$ have to be treated specially, and in fact, the following holds for $P_{-1}(t)=p(0, t)$ :

$$
\frac{d P_{-1}}{d t}(t)=f\left(0, P_{-1}(t), q(0, t), S(0, t), N(t)\right), \quad P_{-1}\left(t_{j-1}\right)=p_{-1}^{j-1},
$$

and the following for $P_{n+1}(t)=p(1, t)$ :

$$
\frac{d P_{n+1}}{d t}(t)=f\left(1, P_{n+1}(t), q(1, t), 0, N(t)\right), \quad P_{n+1}\left(t_{j-1}\right)=p_{n+1}^{j-1} .
$$

So the next time step approximate solution, i.e., the values of the pair $\left(p_{i}^{j}, q_{i}^{j}\right)=$ $\left(p\left(x_{i}, t_{j}\right), q\left(x_{i}, t_{j}\right)\right), i=-1, \ldots, n+1$, except $p_{0}^{j}$, can be approximately computed as the approximate values of $\left(P_{i}\left(t_{j}\right), Q_{i}\left(t_{j}\right)\right)$ by means of a (single step application of an) explicit two stages Runge-Kutta method as the Heun's method or predictor-corrector method. Of course, the computation of the values of $f$ and $g$ involve quadratures (the values of $N\left(t_{j-1}\right)$ and of $S\left(x_{i}, t_{j-1}\right)$ ) which are simply approximated by the trapezoidal rule. The value of $p_{0}^{j}$ is obtained by interpolation using $p_{-1}^{j}$ and $p_{1}^{j}$ since there is no previously computed value of $p$ on the characteristic line through $\left(x_{0}, t_{j}\right)$.

Going to some details, the numerical scheme works as follows. Let us first use the initial conditions to set $p_{i}^{0}=p^{0}\left(x_{i}\right), q_{i}^{0}=q^{0}\left(x_{i}\right), i=-1, \ldots, n+1$. Then, assuming we know $p_{i}^{j-1}$ and $q_{i}^{j-1}$ for $i=-1, \ldots, n+1$ and some $j \geq 1$, we compute the approximate solution for the next time step $j$ in two steps. For the first one, let us set, using the trapezoidal rule,

$$
N^{j-1}=\sum_{i=-1}^{n}\left(\phi\left(x_{i}\right) p_{i}^{j-1}+\psi\left(x_{i}\right) q_{i}^{j-1}+\phi\left(x_{i+1}\right) p_{i+1}^{j-1}+\psi\left(x_{i+1}\right) q_{i+1}^{j-1}\right) \frac{x_{i+1}-x_{i}}{2}
$$

$S_{n+1}^{j-1}=0$, and

$$
S_{i}^{j-1}=2 \sum_{k=i}^{n}\left(\frac{F\left(x_{k}\right) p_{k}^{j-1}}{x_{k}}+\frac{F\left(x_{k+1}\right) p_{k+1}^{j-1}}{x_{k+1}}\right) \frac{x_{k+1}-x_{k}}{2}
$$

for $i=-1, \ldots, n$ (here $\frac{F\left(x_{-1}\right)}{x_{-1}}$ means $\lim _{x \rightarrow 0^{+}} \frac{F(x)}{x}$ ). Then we compute

$$
\begin{aligned}
k_{p,-1}^{j} & =f\left(x_{-1}, p_{-1}^{j-1}, q_{-1}^{j-1}, S_{-1}^{j-1}, N^{j-1}\right), \\
k_{p, i}^{j} & =f\left(x_{i-1}, p_{i-1}^{j-1}, q_{i-1}^{j-1}, S_{i-1}^{j-1}, N^{j-1}\right) \quad i=1, \ldots, n, \\
k_{p, n+1}^{j} & =f\left(x_{n+1}, p_{n+1}^{j-1}, q_{n+1}^{j-1}, S_{n+1}^{j-1}, N^{j-1}\right),
\end{aligned}
$$

and $k_{q, i}^{j}=g\left(x_{i}, p_{i}^{j-1}, q_{i}^{j-1}, N^{j-1}\right)$ for $i=-1, \ldots, n+1$. 
Then we define the "predicted" values for $p$ and $q$ as

$$
\begin{aligned}
p_{-1}^{* j} & =p_{-1}^{j-1}+h k_{p,-1}^{j}, \\
p_{i}^{* j} & =p_{i-1}^{j-1}+h k_{p, i}^{j} \quad i=1, \ldots, n, \\
p_{n+1}^{* j} & =p_{n+1}^{j-1}+h k_{p, n+1}^{j}
\end{aligned}
$$

and $q_{i}^{* j}=q_{i}^{j-1}+h k_{q, i}^{j} \quad i=-1, \ldots, n+1$. As we have already said, the value of $p_{0}^{* j}$ is obtained by interpolation. Namely, $p_{0}^{* j}=p_{-1}^{* j}\left(1-\frac{x_{0}}{x_{1}}\right)+p_{1}^{* j} \frac{x_{0}}{x_{1}}$.

For the second step, almost as above, we set

$$
N^{* j}=\sum_{i=-1}^{n}\left(\phi\left(x_{i}\right) p_{i}^{* j}+\psi\left(x_{i}\right) q_{i}^{* j}+\phi\left(x_{i+1}\right) p_{i+1}^{* j}+\psi\left(x_{i+1}\right) q_{i+1}^{* j}\right) \frac{x_{i+1}-x_{i}}{2},
$$

$S_{n+1}^{* j}=0$, and

$$
S_{i}^{* j}=2 \sum_{k=i}^{n}\left(\frac{F\left(x_{k}\right) p_{k}^{* j}}{x_{k}}+\frac{F\left(x_{k+1}\right) p_{k+1}^{* j}}{x_{k+1}}\right) \frac{x_{k+1}-x_{k}}{2}
$$

for $i=-1, \ldots, n$ (with the same meaning as above of $\frac{F\left(x_{-1}\right)}{x_{-1}}$ ).

Analogously as above we now compute,

$$
k_{p, i}^{* j}=f\left(x_{i}, p_{i}^{* j}, q_{i}^{* j}, S_{i}^{* j}, N^{* j}\right)
$$

for $i=-1, \ldots, n+1$ except $i=0$, and

$$
k_{q, i}^{* j}=g\left(x_{i}, p_{i}^{* j}, q_{i}^{* j}, N^{* j}\right) \quad i=-1, \ldots, n+1 .
$$

Finally we take the following "predicted-corrected" values for $p$ and $q$,

$$
\begin{aligned}
p_{-1}^{j} & =p_{-1}^{j-1}+\frac{h}{2}\left(k_{p,-1}^{j}+k_{p,-1}^{* j}\right), \\
p_{i}^{j} & =p_{i-1}^{j-1}+\frac{h}{2}\left(k_{p, i}^{j}+k_{p, i}^{* j}\right) \quad i=1, \ldots, n, \\
p_{n+1}^{j} & =p_{n+1}^{j-1}+\frac{h}{2}\left(k_{p, n+1}^{j}+k_{p, n+1}^{* j}\right)
\end{aligned}
$$

and $q_{i}^{j}=q_{i}^{j-1}+\frac{h}{2}\left(k_{q, i}^{j}+k_{q, i}^{* j}\right) \quad i=-1, \ldots, n+1$,

and, again by interpolation, $p_{0}^{j}=p_{-1}^{j}\left(1-\frac{x_{0}}{x_{1}}\right)+p_{1}^{j} \frac{x_{0}}{x_{1}}$.

We have performed some tests of validity of the numerical scheme which are developed in the appendix. The first one consists in the comparison between the approximate solution given by the method and the exact solution to a local partial differential system with the same main part (i.e., the part containing the partial derivatives) as System (21). In the second one we compare the approximate 
solution given by the method in the case when there are $x$-independent solutions, i.e., when $L(x) \equiv L_{0}, F(x)=\frac{2 x}{3}$ (since (15) with $\Gamma(s)=s(1-s)$ ), and the initial conditions are $x$-independent with the "exact" solution of System (7).

Applying the numerical scheme to System (2) we observe different kind of behavior depending on the parameter values that we show in Figures 3,4 and 5.

Note that in order to attain a particular final time (different and not an integer multiple of $T$ defined at the beginning of the section) we have modified slightly the construction of the natural grid.
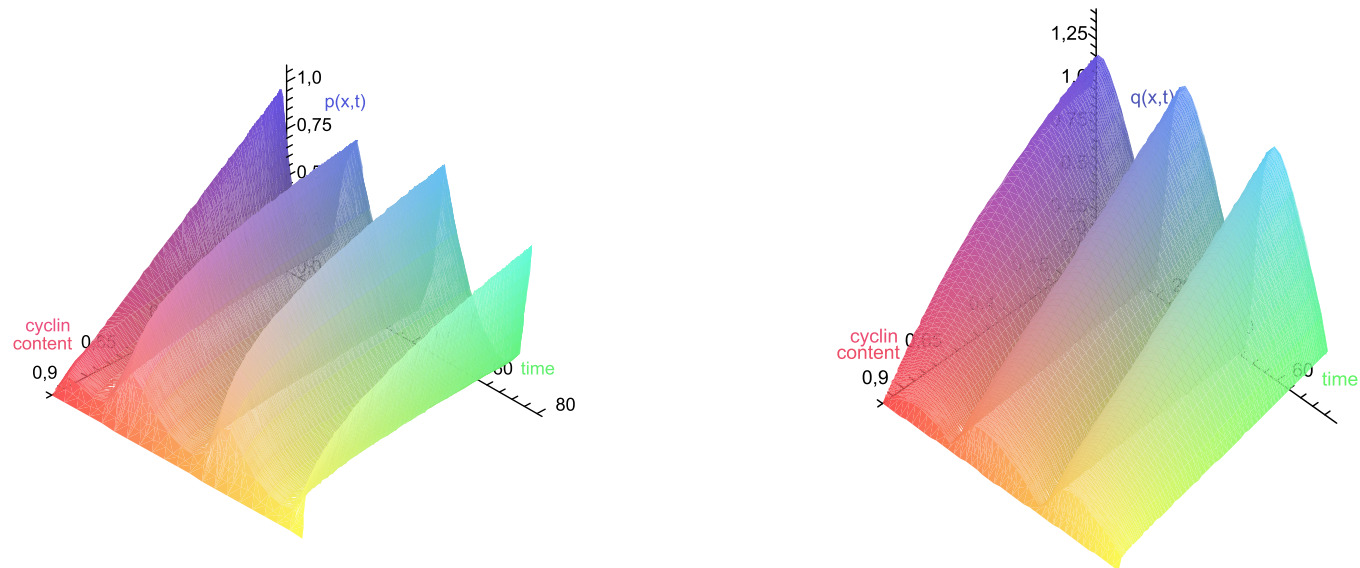

FIgURE 3. Illustration for oscillation behavior with numerical parameters $x_{0}=0.02, T_{f}=80, n=98, m=980, h=0.0816$ and with the model parameters $L(x)=0.4-\frac{x}{4}, F(x)=0.9 x, d_{1}=$ $0.2333, d_{2}=0.12, G(x)=\frac{8}{1+x^{5}}, \phi(x) \equiv 1, \psi(x) \equiv 5$ and $p(x, 0)=$ $1-x, q(x, 0)=1-x^{2}$.

\section{APPENDIX}

The first test that we have performed compares the approximate solution given by the method and the exact solution of the following system

$$
\left\{\begin{array}{l}
\frac{\partial}{\partial t} u(x, t)+\frac{\partial}{\partial x}(x(1-x) u(x, t))=u(x, t)+v(x, t), \\
\frac{\partial}{\partial t} v(x, t)=-v(x, t),
\end{array}\right.
$$

for $t>0$ and $x \in[0,1]$, supplemented by initial conditions of the form $u(x, 0)=$ $u^{0}(x)$ and $v(x, 0)=v^{0}(x)$. System (14) can be obviously reduced to a single p.d.e. of the form

$$
\frac{\partial}{\partial t} u(x, t)+x(1-x) \frac{\partial}{\partial x} u(x, t)=2 x u(x, t)+v^{0}(x) e^{-t}, u(x, 0)=u^{0}(x) .
$$



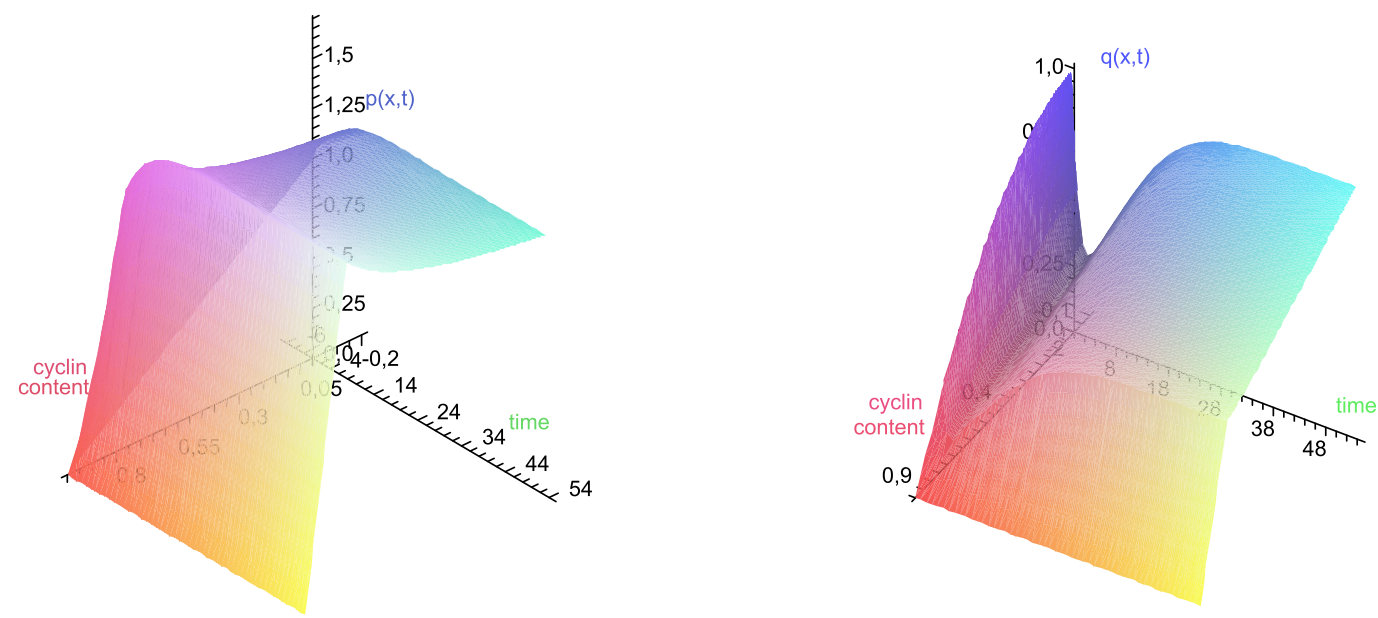

FiguRE 4. Illustration for convergence to equilibria behavior with $x_{0}=0.02, T_{f}=55, n=98, m=980, h=0.056$ and with the parameters $L(x)=0.4-\frac{x}{4}, F(x)=0.6 x, d_{1}=0.1, d_{2}=0.15, G(x)=$ $\frac{5}{1+x^{2}}, \phi(x) \equiv 1, \psi(x) \equiv 3$ and $p(x, 0)=1-x, q(x, 0)=1-x^{2}$.
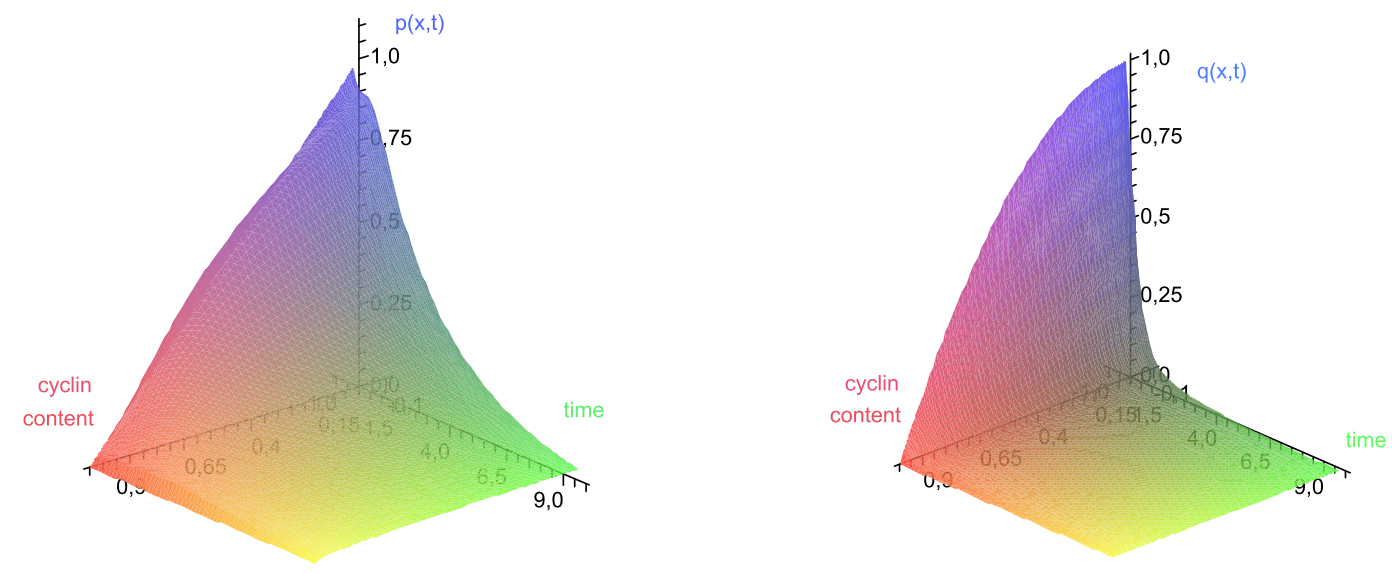

FiguRE 5. Illustration for extinction behavior with $x_{0}=$ $0.02, T_{f}=10, n=100, m=125, h=0.08$ and with the parameters $L(x)=0.4-\frac{x}{4}, F(x)=x, d_{1}=0.7, d_{2}=1, G(x)=\frac{5}{1+x^{2}}, \phi(x) \equiv$ $1, \psi(x) \equiv 5$ and $p(x, 0)=1-x, q(x, 0)=1-x^{2}$.

Assuming that $u^{0}$ and $v^{0}$ are $C^{1}$ functions, the method of characteristics yields an explicit solution of the above problem in the form

$$
\begin{aligned}
& u(x, t)=\left[\frac{e^{t} \Phi(-t, x)}{x}\right]^{2} u_{0}(\Phi(-t, x))+\frac{x e^{-t}}{(1-x)^{3}}[F(x)-F(\Phi(-t, x))], \\
& s \in(0,1), t \in \mathbb{R},
\end{aligned}
$$


where $\Phi(t, x)$ is given, as above, by the solution of the initial value problem

$$
z^{\prime}(t)=\Gamma(z(t)), \quad z(0)=x
$$

and $F$ is a primitive of the function $\left(\frac{1}{y}-1\right)^{2} v^{0}(y)$. This can be checked by means of a straightforward but tedious computation. Obviously, $v(x, t)=v^{0}(x) e^{-t}$.

The numerical scheme for this system has to be adapted somehow and of course it turns to be simpler. In particular, we simply have $f(x, u, v)=2 x u+v$, $g(x, u, v)=-v$.

Table 1 shows the comparative results when one chooses $u^{0}(x)=1-x^{2}$ and $v^{0}(x)=x^{2}$. The column "error" (relative error in $L^{1}$ norm) is evaluated as

$$
\frac{\int_{0}^{1}\left(\left|u_{a}(x, t)-u(x, t)\right| d x+\int_{0}^{1}\left|v_{a}(x, t)-v(x, t)\right| d x\right.}{\int_{0}^{1}(|u(x, t)|+|v(x, t)|) d x},
$$

where the subscript " $a$ " refers to "approximate". Here we consider $T_{f}=1$.

\section{TABLE 1.}

\begin{tabular}{cccccc}
$x_{0}$ & $n$ & $h$ & $m$ & CPU time in seconds & error \\
\hline 0.02 & 117 & 0.0666 & 15 & - & $1.5 \cdot 10^{-3}$ \\
\hline 0.015 & 168 & 0.05 & 20 & 0.5 & $8.7 \cdot 10^{-4}$ \\
\hline 0.01 & 230 & 0.04 & 25 & 1 & $5.5 \cdot 10^{-4}$ \\
\hline 0.005 & 530 & 0.02 & 50 & 4.7 & $1.4 \cdot 10^{-4}$ \\
\hline 0.002 & 1612 & 0.0077 & 130 & 42 & $2.1 \cdot 10^{-5}$ \\
\hline 0.001 & 3519 & 0.00392 & 255 & 228 & $5.6 \cdot 10^{-6}$ \\
\hline
\end{tabular}

The second test that we have performed compares the approximate solution given by the numerical method with the solution of the ordinary differential System (7) for values of the parameters that give $x$-independent solutions and convergence to the steady state. The parameters used are $L(x)=0.4, F(x)=$ $\frac{2 x}{3}, \phi(x)=1, \psi(x)=3, d_{1}=0.1333, d_{2}=0.15, G(N)=\frac{5}{1+N^{2}}$ and the initial conditions $p(x, 0)=1$ and $q(x, 0)=0.7$. The results are shown in Table 2 .

In Table 3 the parameters have been chosen like in Figure 2 in such a way that we compare approximate solutions given by the method and solutions to System (7) in the case of oscillations. The parameters used are $L(x)=0.5, F(x)=$ $\frac{2 x}{3}, \phi(x)=1, \psi(x)=4, d_{1}=0.2333, d_{2}=0.12, G(N)=\frac{6.5}{1+N^{4}}$ and the initial conditions $p(x, 0)=0.25$ and $q(x, 0)=0.45$. 
TABle 2.

\begin{tabular}{ccccccc}
$T_{f}$ & $x_{0}$ & $n$ & $h$ & $m$ & CPU time in seconds & error \\
\hline 1 & 0.1 & 22 & 0.2 & 5 & $<0.1$ & $5 \cdot 10^{-5}$ \\
\cline { 2 - 7 } & 0.05 & 59 & 0.1 & 10 & $<0.1$ & $1.3 \cdot 10^{-5}$ \\
\cline { 2 - 7 } & 0.01 & 230 & 0.04 & 25 & 2.5 & $2 \cdot 10^{-6}$ \\
\cline { 2 - 7 } & 0.002 & 1612 & 0.0077 & 130 & 99 & $5 \cdot 10^{-8}$ \\
\hline 10 & 0.1 & 12 & 0.333 & 30 & 0.2 & $1.7 \cdot 10^{-5}$ \\
\cline { 2 - 7 } & 0.05 & 30 & 0.2 & 50 & 1 & $5.8 \cdot 10^{-5}$ \\
\cline { 2 - 7 } & 0.01 & 234 & 0.0385 & 260 & 18 & $2.1 \cdot 10^{-6}$ \\
\cline { 2 - 7 } & 0.002 & 1554 & 0.00772 & 1295 & 1000 & $1 \cdot 10^{-7}$ \\
\hline 50 & 0.1 & 11 & 0.4545 & 110 & 1 & $8.9 \cdot 10^{-9}$ \\
\cline { 2 - 7 } & 0.05 & 30 & 0.1666 & 300 & 5 & $1.3 \cdot 10^{-9}$ \\
\hline
\end{tabular}

TABLE 3.

\begin{tabular}{ccccccc}
$T_{f}$ & $x_{0}$ & $n$ & $h$ & $m$ & CPU time in seconds & error \\
\hline \multirow{2}{*}{1} & 0.1 & 22 & 0.2 & 5 & $<0.1$ & $5.6 \cdot 10^{-4}$ \\
\cline { 2 - 7 } & 0.01 & 230 & 0.04 & 25 & 4 & $2.4 \cdot 10^{-5}$ \\
\cline { 2 - 7 } & 0.002 & 1612 & 0.0077 & 130 & 122 & $1.7 \cdot 10^{-6}$ \\
\hline \multirow{2}{*}{10} & 0.1 & 12 & 0.333 & 30 & $<1$ & $2.6 \cdot 10^{-3}$ \\
\cline { 2 - 7 } & 0.01 & 234 & 0.0385 & 260 & 37 & $4 \cdot 10^{-5}$ \\
\hline 50 & 0.1 & 11 & 0.4545 & 110 & 1 & 0.02 \\
\cline { 2 - 7 } & 0.05 & 30 & 0.1666 & 300 & 7 & $3.3 \cdot 10^{-3}$ \\
\hline
\end{tabular}

\section{REFERENCES}

[1] L. Abia, O. Angulo, J.C. López-Marcos, M.A. López-Marcos, Numerical schemes for a sizestructured cell population model with equal fission, Mathematical and Computer Modelling, 50, (2009) 653-664.

[2] M. Adimy, L. Pujo-Menjouet, Asymptotic behavior of a singular transport equation modelling cell division, DCDS(B) 3(3) (2003), 439-456.

[3] O. Angulo, J. C. López-Marcos, Numerical schemes for size-structured population equations, Math. Biosci. 157(1-2) (1999), 169-188.

[4] O. Arino, A survey of structured cell population dynamics. Acta Biotheoret, 43 (1995) $3-25$.

[5] O. Arino, E. Sánchez, G.F. Webb, Necessary and sufficient conditions for asynchronous exponential growth in age structured cell populations with quiescence. J. Math. Anal. Appl. 215(2) (1997), 499-513. 
[6] F. Bekkal Brikci, J. Clairambault, B. Ribba and B. Perthame, An age-and-cyclin-structured cell population model with proliferation and quiescence, J. Math. Biol. 57(1) (2008), 91-110.

[7] F. Bekkal Brikci, J. Clairambault and B. Perthame, Analysis of a molecular structured population model with possible polinomial growth for the cell division cycle, Math. Comput. Model. 47(7-8) (2008), 699-713.

[8] G. Bell, E. Anderson, Cell growth and division. Mathematical model with applications to cell volume distributions in mammalian suspension cultures. Biophys. J. 7 (1967), 329-351.

[9] R. Borges, À. Calsina, S. Cuadrado. Equilibria of a cyclin structured cell population model Discrete and Continuous Dynamical Systems - Series B, 11 (2009), 613-627.

[10] M. Doumic, Analysis of a population model structured by the cells molecular content, Math. Model. Nat. Phenom. 2(3) (2007), 121-152.

[11] J. Dyson, R. Vilella-Bressan and G. Webb, Asynchronous exponential growth in an age structured population of proliferating and quiescent cells, Math. Biosc. 177/178 (2002), 73-83.

[12] M. Gyllenberg and G. Webb, Age-size structure in populations with quiescence, Math. Biosci. 86(1) (1987), 67-95.

[13] J.A.J. Metz and O. Diekmann, The dynamics of physiologically structured populations (Amsterdam, 1983), 136-184, Lecture Notes in Biomath, no. 68, Springer, Berlin, 1986.

[14] H. Von Foerster, Some remarks on changing populations, "The kinetics of cellular proliferation" (Frederick Stohlman, ed.), Grune and Stratton, New York, 1959, 382-407.

[15] L.H. Hartwell, M.B. Kastan, Cell cycle control and cancer, Science 266 (1994), 1821-1828.

[16] T. Kostova, An explicit third-order numerical method for size-structured population equations, Numer. Methods Partial Differential Equations 19(1) (2003), 1-21.

[17] B. Rossa, Asynchronous exponential growth in a size structured cell population with quiescent compartment, in: O. Arino, D. Axelrod, M. Kimmel (Eds.), Mathematical Population Dynamics, vol. 2, Wuerz, Canada, 1995.

RICARDO BORGES

Departament de Matemàtiques

Universitat Autònoma de Barcelona

08193 Bellaterra (Barcelona), Spain

E-mail address: rborges@mat.uab.cat

Àngel Calsina

Departament de Matemàtiques

Universitat Autònoma de BARCELONA

08193 Bellaterra (BArcelona), Spain

E-mail address: acalsina @mat.uab.cat

Sílvia Cuadrado

Departament de Matemàtiques

Universitat Autònoma de BARCELONA

08193 Bellaterra (BARCElona), SPAin

E-mail address: silvia@mat.uab.cat 